\title{
Adenocarcinoma de pulmón que simula tuberculosis miliar
}

\author{
Dawlat Khan $^{\text {a }}$ Mohammed Danjuma ${ }^{a}$ Muhammad Umar Saddique ${ }^{a}$ \\ Khaled A. Murshed ${ }^{\mathrm{b}}$ Mohamed A. Yassin ${ }^{\mathrm{c}}$ \\ ${ }^{a}$ Departamento de Medicina, Hospital General Hamad - Corporación Médica Hamad Qatar, Doha, Qatar; \\ ${ }^{b}$ Departamento de Patología, Hospital General Hamad - Corporación Médica Hamad Qatar, Doha, Qatar; \\ 'Departamento de Oncología-Hematología Médica, Corporación Médica Hamad Qatar, Doha, Qatar
}

\author{
Palabras clave \\ Sombras/patrón radiológico miliar · Tuberculosis miliar . \\ Adenocarcinoma $\cdot$ Metástasis intrapulmonares
}

\section{Resumen}

Las sombras miliares en imágenes del tórax tienen diagnósticos diferenciales muy diversos. La etiología más común es infecciosa, como la tuberculosis (TB) miliar y la histoplasmosis, pero las sombras miliares pueden ser la presentación de la sarcoidosis, neumoconiosis y metástasis secundaria al pulmón de cánceres primarios de tiroides, riñón y trofoblastos, así como del sarcoma. Presentamos el caso de un paciente indio, masculino, quien refirió una historia de dos meses con tos seca y dificultad respiratoria. Imágenes del tórax mostraron nódulos miliares bilaterales, difusos. La impresión inicial fue TB pulmonar miliar. Una posterior broncoscopía con biopsia transbronquial confirmó el diagnóstico de adenocarcinoma mucinoso pulmonar con metástasis al cerebro, una presentación rara e inusual de cáncer de pulmón primario. El tumor fue positivo para ALK5A4 y PD-L1, y se inició inmunoterapia con un inhibidor de tirosina quinasa, con respuesta favorable.

(C) 2020 El (los) autor(es). Publicado por S. Karger AG, Basilea

\section{Introducción}

Las sombras miliares en los pulmones se presentan en una amplia variedad de condiciones. El patrón radiológico miliar se observa como numerosos nódulos pequeños (usualmente, de 1-3 mm de diámetro) en el pulmón, con márgenes definidos [1].

El patrón miliar en las radiografías del tórax podría ser debido a la tuberculosis (TB) miliar, histoplasmosis, sarcoidosis, neumoconiosis, carcinoma broncoalveolar o siderosis pulmonar. También puede ser signo de metástasis secundaria a los pulmones a partir de cáncer primario de tiroides, riñón y trofoblastos, así como algunos sarcomas $[2,3]$.

El cáncer de pulmón se encuentra entre los cánceres de diagnóstico más frecuente, y es la causa principal de muerte relacionada con el cáncer [4]. Los cánceres de pulmón se clasifican en cáncer pulmonar microcítico (CPM) y no microcítico (CPNM). El CPNM se divide a su vez en tres subtipos principales: carcinoma de células escamosas, adenocarcinoma y carcinoma de células grandes. El adenocarcinoma de pulmón es el tipo más común entre no fumadores y mujeres $[4,5]$.

Típicamente, los adenocarcinomas pulmonares surgen del epitelio bronquial o del epitelio bronquial glandular. Patológicamente, el adenocarcinoma puede exhibir un patrón de crecimiento acinar, papilar, micropapilar, lepídico o sólido, presenta expresión de marcadores mucinosos o neumocíticos, y usualmente se localiza en la periferia, con afectación pleural [6]. Los hallazgos radiológicos más frecuentes consisten en nódulos con aspecto de cristal molido, parcialmente sólidos, o sólidos en tomografía computarizada (TC) del tórax [7]. El cáncer pulmonar primario rara vez se presenta en forma de nódulos miliares [8-10]. Aquí presenta- information@karger.com www.karger.com/kxn

Karger ${ }^{\prime \prime}=$ $\begin{array}{ll}\text { (C) } 2020 \mathrm{El} \text { (los) autor(es) } & \text { Karger } \\ \text { Publicado por S. Karger AG, Basilea } & \text { Open ciccess }\end{array}$

This article is licensed under the Creative Commons Attribution 4.0 International License (CC BY) (http://www.karger.com/Services/Open AccessLicense). Usage, derivative works and distribution are permitted provided that proper credit is given to the author and the original publisher.
Dr. Dawlat Khan

Residente en Medicina Interna, Departamento de Medicina

Hospital General Hamad, Corporación Médica Hamad Qatar

Doha 3050, Qatar

drhafiz2012@gmail.com 


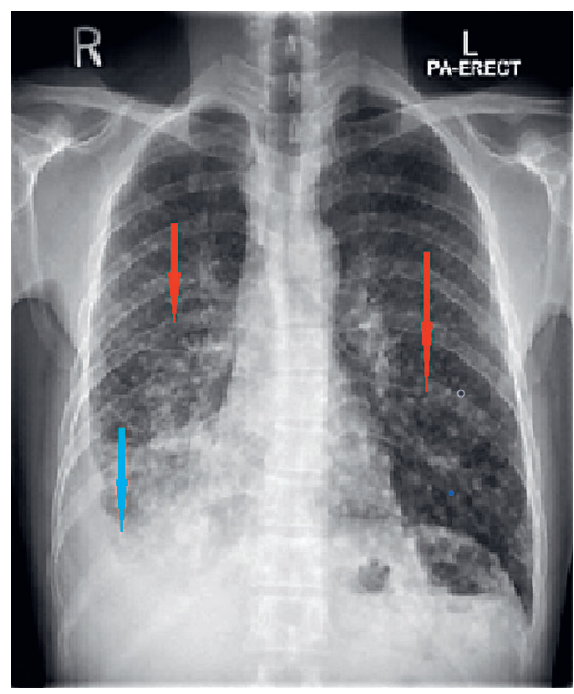

Fig. 1. Rayos $X$ del tórax. Vista posterior-anterior que muestra sombras miliares bilaterales (flechas rojas) y efusión pleural en el lado derecho (flecha azul).

mos el caso de un paciente masculino, no fumador, de 35 años, procedente de un área endémica para $\mathrm{TB}$, con un moteo miliar en los rayos $\mathrm{X}$ del tórax, y diagnosticado con adenocarcinoma pulmonar.

\section{Presentación del caso}

Un paciente masculino de 35 años, procedente de una región endémica para TB, se presentó en el Departamento de Emergencias con una historia de dos meses con tos seca, dificultad respiratoria y dolor en el lado derecho del tórax. Tuvo dos episodios de hemoptisis con pequeñas cantidades de sangre una semana antes de acudir al hospital. No tenía historia de fiebre o sudores nocturnos y había perdido alrededor de 2-3 kg durante los dos meses anteriores.

El paciente no tenía historia de contacto cercano con TB ni de viaje reciente. No es fumador, no tiene historia médica ni quirúrgica previa significativa, y trabaja como carpintero. Tampoco había antecedentes de comportamiento sexual de alto riesgo ni de drogadicción.

Los signos vitales del paciente y los resultados del examen físico fueron benignos, excepto por una nota sorda a la percusión en el tórax inferior derecho y una reducción en la entrada de aire a la auscultación. Los análisis de laboratorio revelaron un nivel de hemoglobina de $14 \mathrm{~g} / \mathrm{dL}$ (intervalo de referencia, 13.0-17.0) y un conteo total de leucocitos de $9,300 / \mathrm{mm} 3$ (intervalo de referencia, 4.0-10.0 × 109/L; adición/corrección del editor al artículo original), aunque los conteos diferenciales fueron normales. Los resultados de las pruebas de función hepática y renal se encontraron entre límites normales. El nivel de proteína C-reactiva fue 22 $\mathrm{mg} / \mathrm{L}$ (intervalo de referencia, $0-5$ ). Los rayos X del tórax mostraron extensos infiltrados pulmonares bilaterales con un patrón miliar, y efusión pleural en el lado derecho (Fig. 1). Inicialmente

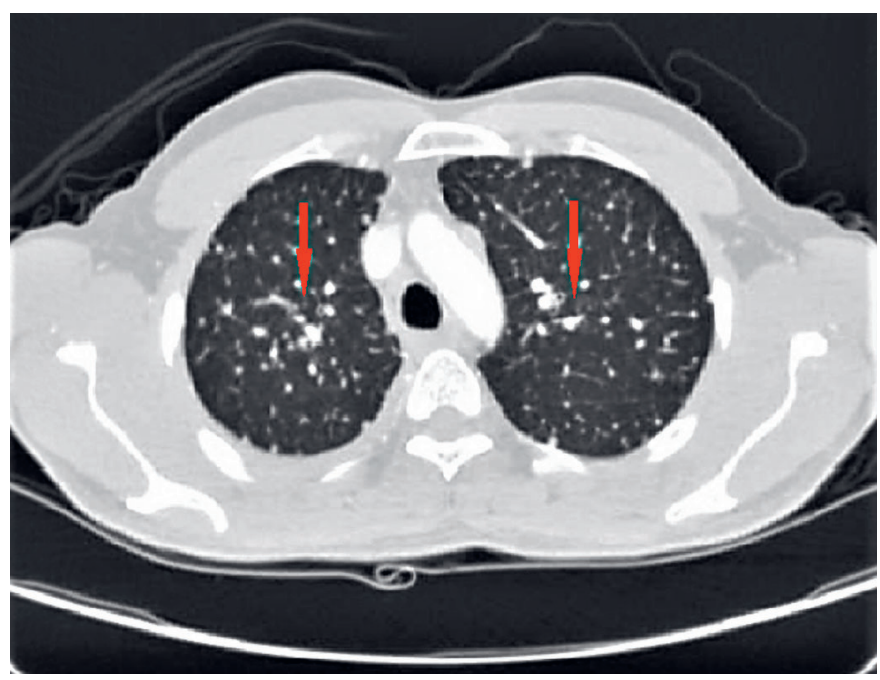

Fig. 2. TC del tórax que muestra infiltrados nodulares miliares bilaterales (flechas rojas).

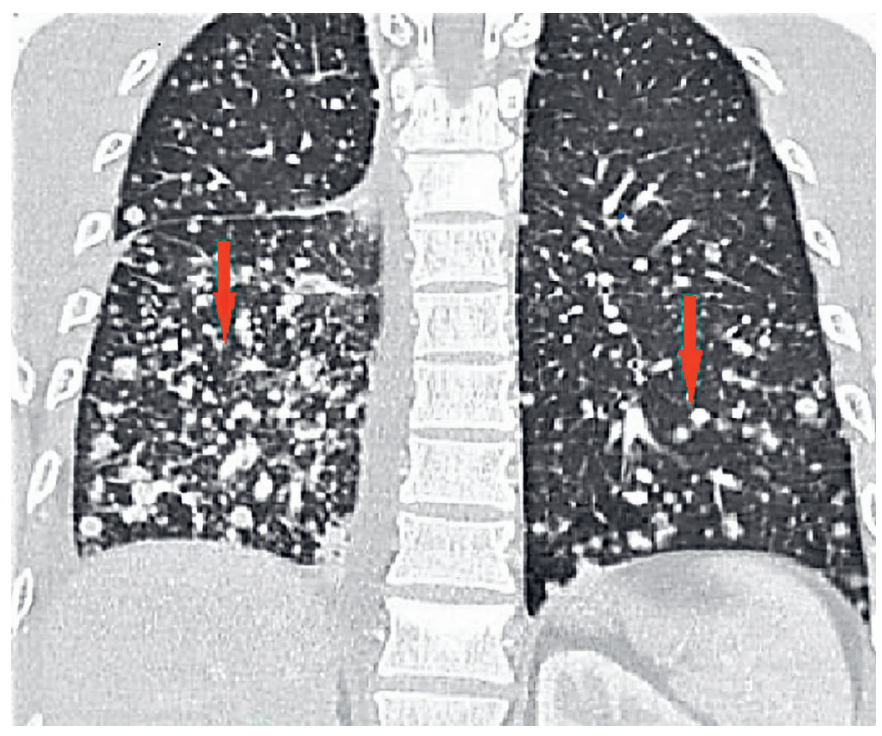

Fig. 3. TC del tórax que muestra múltiples nódulos miliares bilaterales (flechas rojas).

se tomaron precauciones contra la posible diseminación aérea del agente infeccioso. Las siguientes pruebas, que se practicaron para limitar el diagnóstico diferencial, dieron resultados negativos: ensayo QuantiFERON-TB Gold, tinción para bacilos acidorresistentes en frotis de esputo (dos muestras), prueba cutánea de tuberculina, ensayo de anticuerpos contra el virus de la inmunodeficiencia humana y tamiz de neumonitis por hipersensibilidad.

Una TC del tórax confirmó la presencia de múltiples infiltrados nodulares miliares distribuidos en ambos pulmones, linfadenopatía mediastinal, y una lesión en los tejidos blandos del hilio derecho, así como múltiples lesiones densas en la espina dorsal y linfadenopatía retroperitoneal abdominal (Fig. 2, 3). Una tomografía por emisión de positrones (PET) de cuerpo entero confirmó la presencia de un tumor primario en el pulmón derecho con extensas metástasis pulmonares en forma de nódulos pequeños, 


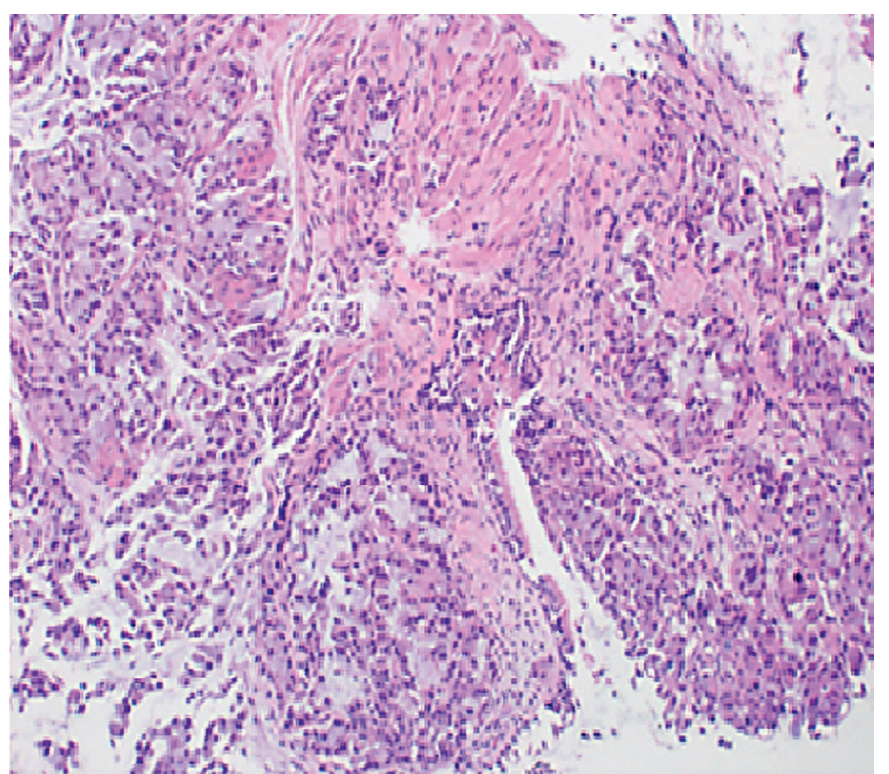

Fig. 4. Biopsia transbronquial que muestra el tumor con un patrón de crecimiento acinar. H\&E. $\times 200$.

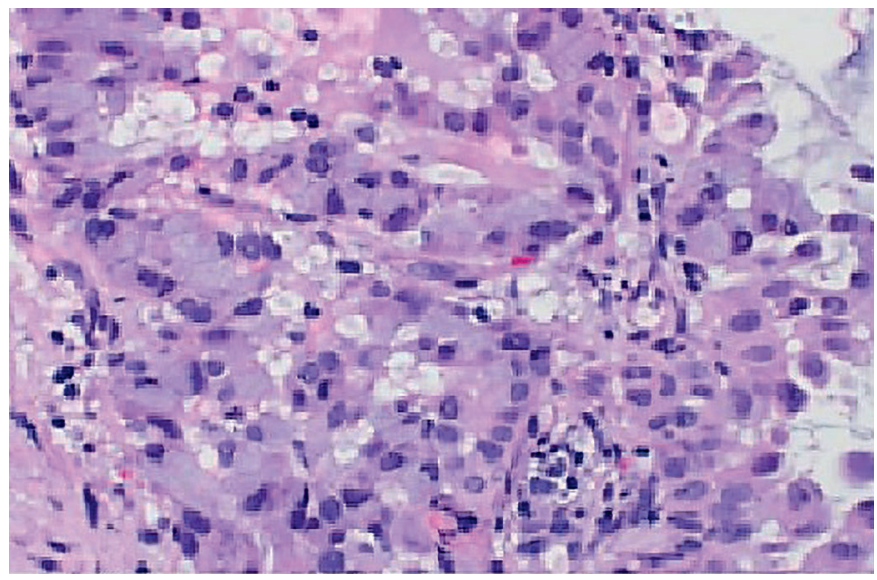

Fig. 5. Células tumorales que muestran atipia celular, con núcleos prominentes y abundante mucina intracitoplásmica. H\&E. ×400.

y metástasis hiliares bilaterales, múltiples metástasis mediastinales en la parte inferior izquierda del cuello y en los nódulos linfáticos retroperitoneales superiores y paraaórticos, así como múltiples metástasis escleróticas en los huesos. Una resonancia magnética nuclear (RMN) de la cabeza reveló múltiples (alrededor de 5) focos de realce anormal, distribuidos en ambos hemisferios cerebrales pero principalmente en el lado izquierdo, probablemente metastásicos.

Una broncoscopía de video con fibra óptica flexible no mostró características notables en las cuerdas vocales, la tráquea y carina, y el pulmón izquierdo. El pulmón derecho mostró infiltración nodular a partir del bronquio principal derecho y extendida hacia el bronquio intermedio derecho, causando la obstrucción completa del segmento apical del lóbulo inferior derecho. Se practicó un lavado broncoalveolar, un cepillado bronquial y biopsias transbronquiales guiadas por fluoroscopio de las masas del lado derecho.

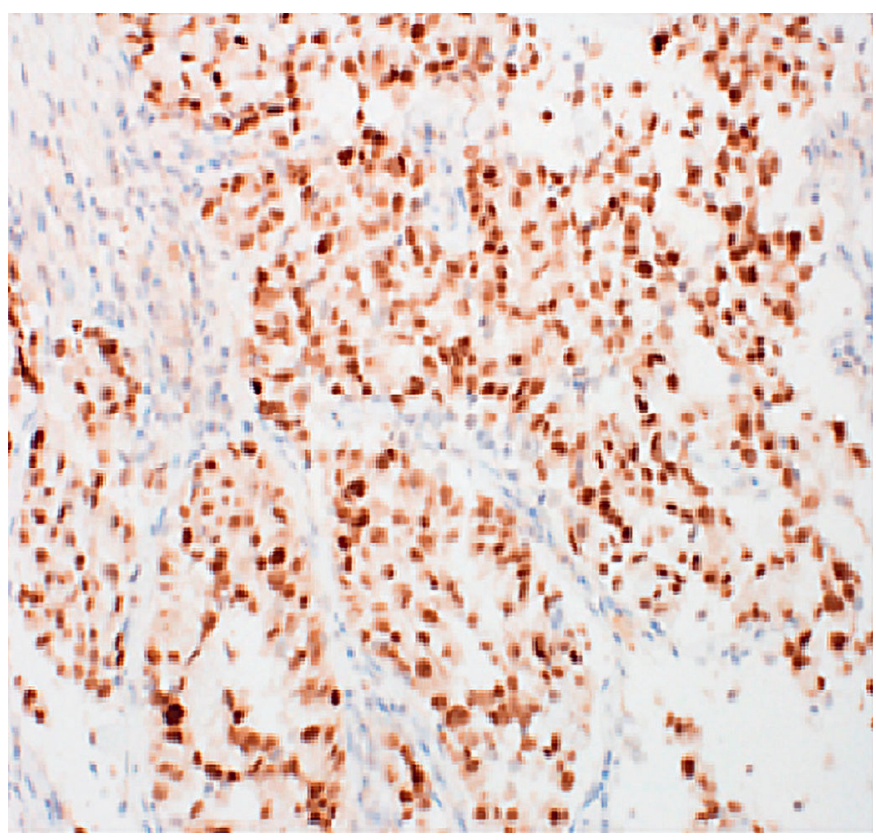

Fig. 6. Células tumorales que muestran una intensa tinción nuclear difusa para TTF-1.

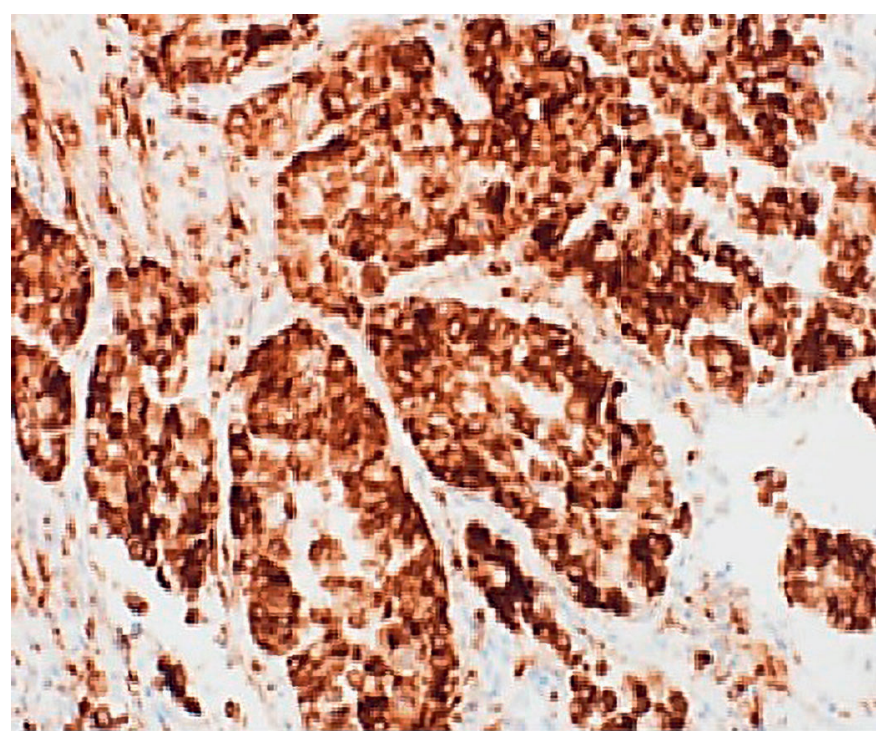

Fig. 7. Intensa tinción citoplásmica difusa para napsin A

El lavado broncoalveolar resultó negativo para células malignas, y el examen histopatológico indicó un adenocarcinoma mucinoso pulmonar (Fig. 4-6). En el análisis inmunohistoquímico, las células tumorales fueron positivas para napsin A (Fig. 7) y para la quinasa de linfoma anaplásico (ALK5A4) (Fig. 8), y fuertemente positivas para el ligando 1 de muerte programada (PD-L1) (Fig. 9). La mutación EGFR fue negativa.

El paciente se remitió a un oncólogo y se inició tratamiento con inhibidores de tirosina quinasa, inicialmente crizotinib y después alectinib, con una respuesta favorable. Una TC PET tomada cuatro meses después mostró una resolución notable del nódulo hipermetabólico en el lóbulo inferior del pulmón derecho, así como 


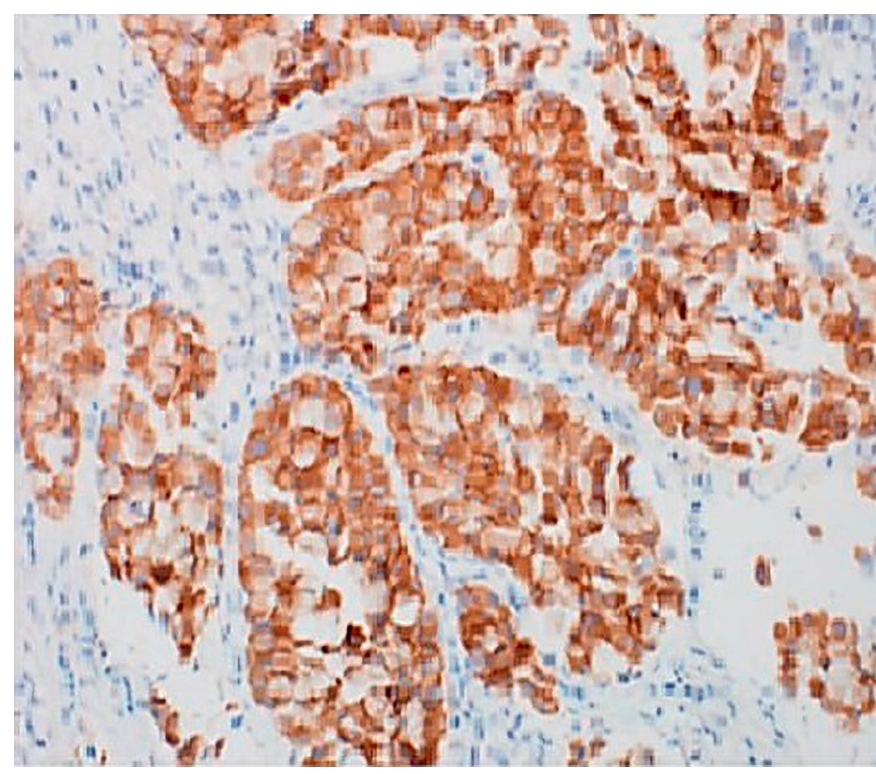

Fig. 8. Intensa tinción citoplásmica difusa para ALK-1.

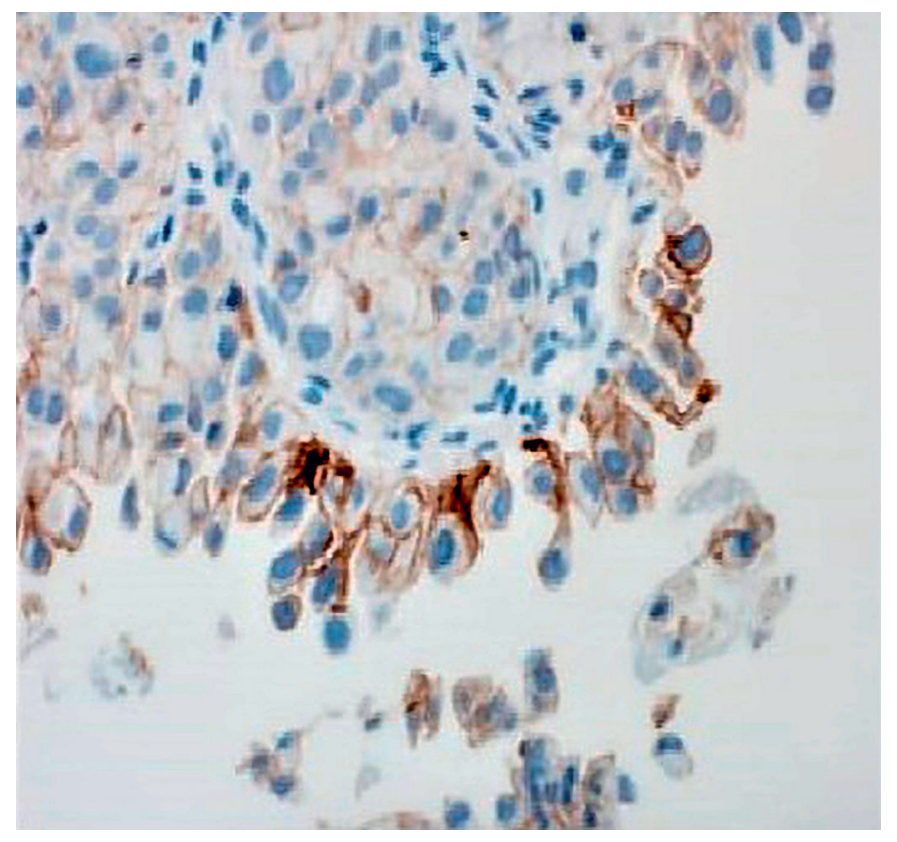

Fig. 9. Tinción membranal moderada a intensa para PD-L1.

una resolución de la efusión pleural y la linfadenopatía en el abdomen y el retroperitoneo. Se observó la resolución metabólica de las lesiones hipermetabólicas en los huesos, y no hubo nuevas lesiones. Una segunda RMN de la cabeza mostró una respuesta favorable al tratamiento. El paciente aún está bajo tratamiento activo, y está tolerando bien la terapia.

\section{Discusión}

El cáncer de pulmón se encuentra entre los tipos de cáncer diagnosticados con mayor frecuencia, y es la principal causa de muerte relacionada con el cáncer en el mundo [4]. La edad de máxima incidencia para el cáncer pulmonar se encuentra entre 55 y 65 años, y el tabaquismo es un factor de riesgo bien establecido: los fumadores activos y pasivos tienen un riesgo 13 y 1.5 veces mayor que los no fumadores, respectivamente [11]. Los cánceres de pulmón se clasifican en CPM y CPNM. El CPNM se clasifican a su vez en tres subtipos principales: carcinoma de células escamosas, adenocarcinoma y carcinoma de células grandes $[4,5]$. Con frecuencia, el carcinoma de células escamosas y el CPM se presentan en forma de masa central [12].

Típicamente, los adenocarcinomas pulmonares surgen del epitelio bronquial o del epitelio glandular bronquial, y constituyen el tipo más común entre no fumadores y mujeres $[4,5]$. Patológicamente, el adenocarcinoma puede exhibir un patrón de crecimiento acinar, papilar, micropapilar, lepídico o sólido, con expresión de marcadores mucinosos o neumocíticos, y usualmente se localiza en la periferia, con afectación a la pleura [6]. El hallazgo radiológico más frecuente en TC de tórax es la presencia de nódulos con aspecto de cristal molido, parcialmente sólido, o sólido [7]. El cáncer pulmonar primario rara vez se presenta como nódulos miliares [8-10]. Las sombras miliares en los pulmones se presentan en una amplia variedad de condiciones. El patrón miliar adquiere la forma de numerosos nódulos (usualmente de $1-3 \mathrm{~cm}$ de diámetro) en el pulmón, que suelen mostrar márgenes definidos [1]. El patrón miliar en la radiografía del tórax podría ser una presentación de TB miliar, histoplasmosis, sarcoidosis, neumoconiosis, carcinoma broncoalveolar o siderosis pulmonar. También puede ser signo de metástasis secundaria a los pulmones de cánceres primarios en tiroides, riñón o trofoblastos, así como algunos sarcomas [2, 3]. Una TC de alta resolución puede ayudar a reducir los diagnósticos diferenciales, al clasificar los nódulos en patrones centrolobulillares, perilinfáticos y aleatorios. La bronquiolitis infecciosa comúnmente presenta un patrón centrolobulillar, y la sarcoidosis un patrón perilinfático, mientras que un patrón aleatorio es sugestivo de metástasis hematógena, como en nuestro paciente (Fig. 3) [12]. Una revisión exhaustiva de la literatura reveló algunos casis similares, reportados como carcinoma miliar primario de pulmón [2]. La serie de casos más extensa se ha reportado en Japón, e incluyó cinco pacientes [3]. Ese trabajo concluyó que la prevalencia del fenotipo miliar es $<1 \%$ en pacientes japoneses, y tiene mal pronóstico.

El adenocarcinoma es el tipo más común de cáncer pulmonar entre no fumadores y mujeres $[4,5]$. En áreas endémicas para TB, siempre es tentador tratar a pacientes que presenten sombras miliares en imágenes torácicas como casos de TB miliar [13]. La presentación del adenocarcinoma pulmonar como sombras miliares es rara, pero tiene mal pronóstico si se retrasa el diagnóstico. La inmunoterapia con inhibidores de tirosina quinasa e inhibidores de fusión de la quinasa de linfoma anaplásico han suscitado respuestas favorables al tratamiento y prolongado la supervivencia total, como en nuestro caso $[11,12]$.

\section{Conclusiones}

Las sombras miliares en las imágenes del tórax podrían indicar la presencia de condiciones comunes como la TB miliar, infecciones 
fúngicas, sarcoidosis y enfermedad pulmonar ocupacional. También pueden ser manifestación de metástasis hematógena a los pulmones a partir de cáncer tiroideo o renal, sarcomas, etc. El adenocarcinoma pulmonar, aunque rara vez presenta un patrón miliar debido a la naturaleza intrapulmonar de sus metástasis, podría pasarse por alto si no se toma una biopsia de tejido y los pacientes reciben tratamiento empírico en áreas endémicas para la TB con base en un diagnóstico radiológico de TB miliar. Esto podría retrasar el diagnóstico y el inicio de un tratamiento basado en inhibidores de tirosina quinasa, lo que conduciría a un mal resultado. Concluimos que el adenocarcinoma pulmonar puede considerarse un diagnóstico diferencial raro pero ominoso de sombras miliares en imágenes del tórax. Tiene una respuesta favorable a la terapia con inhibidores de tirosina quinasa, aun con metástasis al cerebro.

\section{Agradecimientos}

Los autores agradecen el apoyo del Departamento de Patología, Hospital General Hamad Qatar, por proporcionar las imágenes de histopatología, y al Departamento de Educación Médica, Corporación Médica Hamad Qatar, por proporcionar la información médica básica del paciente.

\section{Declaración ética}

Se obtuvo consentimiento de todos los participantes en este estudio. El Comité Revisor del Consejo de Investigación Médica, Corporación Médica Hamad Qatar, revisó y aprobó el protocolo a condición de que no se mencionara información personal alguna en la publicación.

\section{Disclosure Statement}

Todos los autores declaran que no existe potencial conflicto de interés relacionado con la publicación de este reporte de caso.

\section{Fuentes de financiamiento}

La Biblioteca Nacional de Qatar (QNL) Doha, Qatar, cubrió los costos de publicación.

\section{Contribuciones de los autores}

D.K. concibió y planeó la idea, la revisión de la literatura, la recolección de datos y figuras radiológicas, escribió el manuscrito y organizó el reporte de caso; M.D. contribuyó con la revisión de la literatura y colaboró en la escritura del manuscrito; M.U.S. contribuyó con la recolección de datos y figuras radiológicas y colaboró en la escritura del manuscrito; K.A.H.M. contribuyó con la sección de patología del reporte de caso y la revisión de la literatura relacionada; M.A.Y. supervisó el proyecto y la edición del manuscrito.

\section{Información de licencias}

Este artículo se publica bajo la Licencia Internacional Creative Commons Attribution-NonCommercial 4.0 (CC BY-NC) (http://www.karger.com/Services/OpenAccessLicense). El uso y la distribución para propósitos comerciales requiere permiso por escrito.

\section{Referencias}

1 Njeh H, Abid H, Bradaii M, et al.: Miliary disease of the lung: what is your diagnosis? Eur Respir J. 2013;42(Suppl 57):P2260.

2 Eisenberg RL: Clinical imaging: an atlas of differential diagnoses, ed 2. Gaithersburg, MD: Aspen Publishers, 1992, pp 56-59.

3 Koutsopoulos AV, Mitrouska I, Dambaki KI, et al.: Is a miliary chest pattern always indicative of tuberculosis or malignancy? Respiration. 2006;73(3):379-381.

4 Torre LA, Bray F, Siegel RL, et al.: Global cancer statistics, 2012. CA Cancer J Clin. 2015; 65(2):87-108

5 Couraud S, Zalcman G, Milleron B, et al.: Lung cancer in never smokers - a review. Eur J Cancer. 2012;48(9):1299-1311.
6 Pillai S, Khan A, Khan S: Adenocarcinoma of the lung presenting with intrapulmonary miliary metastasis. Cureus. 2019;11(8):e5430.

7 Diederich S, Wormanns D, Semik M, et al.: Screening for early lung cancer with low-dose spiral CT: prevalence in 817 asymptomatic smokers. Radiology. 2002;222(3):773-781.

8 Henschke CI, McCauley DI, Yankelevitz DF, et al.: Early Lung Cancer Action Project: overall design and findings from baseline screening. Lancet. 1999;354(9173):99-105.

9 Chan A, Devanand A, Low SY, et al.: Radial endobronchial ultrasound in diagnosing peripheral lung lesions in a high tuberculosis setting. BMC Pulm Med. 2015;15:90.

10 Fachinger P, Tini GM, Grobholz R, et al.: Pulmonary tularaemia: all that looks like cancer is not necessarily cancer - case report of four consecutive cases. BMC Pulm Med. 2015;15:27.
11 Jayaram Subhashchandra B, Ismailkhan M, Chikkaveeraiah Shashidhar K, et al.: A rare case of non-small cell carcinoma of lung presenting as miliary mottling. Iran J Med Sci. 2013;38(1):65-68.

12 Kimmig L, Bueno J: Miliary nodules: not always tuberculosis. Ann Am Thorac Soc. 2017; 14(12):1858-1860.

13 Prajapat DK, Khanna A, Talwar D: Adenocarcinoma of the lung masquerading as miliary mottling: a rare presentation. Egypt J Intern Med. 2015;27(3):118-120. 